\title{
Apraxia of Eyelid Opening After Brain Injury: A Case Report
}

\author{
Min Jeong Kim, MD, Soo Jin Kim, MD, Bo-Ram Kim, MD, Jongmin Lee, MD, PhD
}

Department of Rehabilitation Medicine, Konkuk University School of Medicine and Konkuk University Medical Center, Seoul, Korea

\begin{abstract}
Apraxia of eyelid opening (AEO) is a syndrome characterized by the patient's difficulty in initiating eyelid elevation spontaneously. Most of the reported cases were associated with extrapyramidal diseases. We report a case of AEO presented after traumatic brain injury, not with extrapyramidal diseases, and improved by dopaminergic treatment. A 49-year-old man underwent a traffic accident and was transferred to the emergency room in an unconscious state. Brain computed tomography (CT) revealed a subdural and epidural hemorrhage at right temporal and bilateral frontal lobes, and he received burr-hole trephination. After receiving comprehensive treatment including occupational therapy, cognition and mobility gradually improved, but he could not open his eyes voluntarily. With dopaminergic treatment (levodopa/benserazide 200/50 mg), he started to open his eyes spontaneously, especially when eating and undergoing physical training. This case showed that AEO may occur after brain injury and that dopaminergic treatment is beneficial also in AEO patients without extrapyramidal diseases.
\end{abstract}

Keywords Apraxia of eyelid opening, Brain injury, Agents, Dopaminergic

\section{INTRODUCTION}

The term 'apraxia of eyelid opening' was coined by Goldstein and Cogan [1] in 1965 to describe a "nonparalytic motor abnormality characterized by the patient's difficulty in initiating the act of lid elevation". In other words, apraxia of eyelid opening (AEO) is a disabling syndrome characterized by an inability to initiate lid open-

Received February 7, 2014; Accepted April 8, 2014

Corresponding author: Jongmin Lee

Department of Rehabilitation Medicine, Konkuk University Medical Center, 120 Neungdong-ro, Gwangjin-gu, Seoul 143-729, Korea

Tel: +82-2-2030-5345, Fax :+82-2-2030-5379, E-mail: leej@kuh.ac.kr

(c) This is an open-access article distributed under the terms of the Creative Commons Attribution Non-Commercial License (http://creativecommons. org/licenses/by-nc/3.0) which permits unrestricted noncommercial use, distribution, and reproduction in any medium, provided the original work is properly cited.

Copyright (C) 2014 by Korean Academy of Rehabilitation Medicine ing voluntarily despite intact frontalis muscle contraction and absence of ocular motor, ocular sympathetic nerve dysfunction, and ocular myopathy. AEO is an uncommon disorder, and its pathogenic mechanism and anatomical site of the decisive lesion have not been fully elucidated with only a handful of AEO cases having been reported abroad so far. Goldstein and Cogan [1] reported the difficulty in initiating the act of lid elevation in four patients with basal ganglia disease, and Riese [2] described AEO in a patient with a frontotemporal injury. Smith et al. [3] reported that AEO was associated with medial frontal hypometabolism. Thereafter, most of the cases reported have been associated with extrapyramidal diseases, such as Parkinson disease, progressive supranuclear palsy, and corticobasal degeneration. Even though a number of case reports have been published on the effectiveness 
of levodopa in a patient with AEO abroad [4-7], there has been no case report so far on AEO and its treatment in Korea. Therefore, we report a patient presented with AEO after acute traumatic brain injury without involving the extrapyramidal motor system and discuss the possible effectiveness of the dopaminergic treatment in improving the symptom.

\section{CASE REPORT}

A 49-year-old and otherwise healthy man underwent a traffic accident and was transferred to emergency room in an unconscious state. Brain computed tomography (CT) revealed traumatic subdural and epidural hemorrhage at the bilateral frontal lobes and right temporal lobe. No abnormality in the extrapyramidal motor system including the basal ganglia was present (Fig. 1). The initial Glasgow Coma Scale (GCS) score was 5 (eye opening response $[\mathrm{E}]=1$, verbal response $[\mathrm{V}]=0$, motor response
$[\mathrm{M}]=4)$. After receiving burr-hole trephination and hematoma drainage, the patient was reevaluated to have a GCS score of 11 (E1, V5, M5). Although verbal and motor responses improved after surgery, he was unable to open his eyes even with a strong painful stimulus. In spite of gradual recovery of mental status and motor function, voluntary eye opening was still not observed. After being transferred to the rehabilitation unit, the patient was examined for the neurological function with mental status being alert and the Mini-Mental State Examination score at 7 . He exhibited left side weakness that was graded fair to good in a manual muscle test. The pupils were $3 \mathrm{~mm}$ in diameter, symmetrical, and equally reactive with extraocular movements fully intact. With the patient unable to open his eyes on command, an examiner elevated the patient's eyelid and found no abnormality in the ocular motor function. No evidence of other apraxia, such as buccofacial and limb apraxia, was present. He received comprehensive rehabilitation treatment, but AEO did
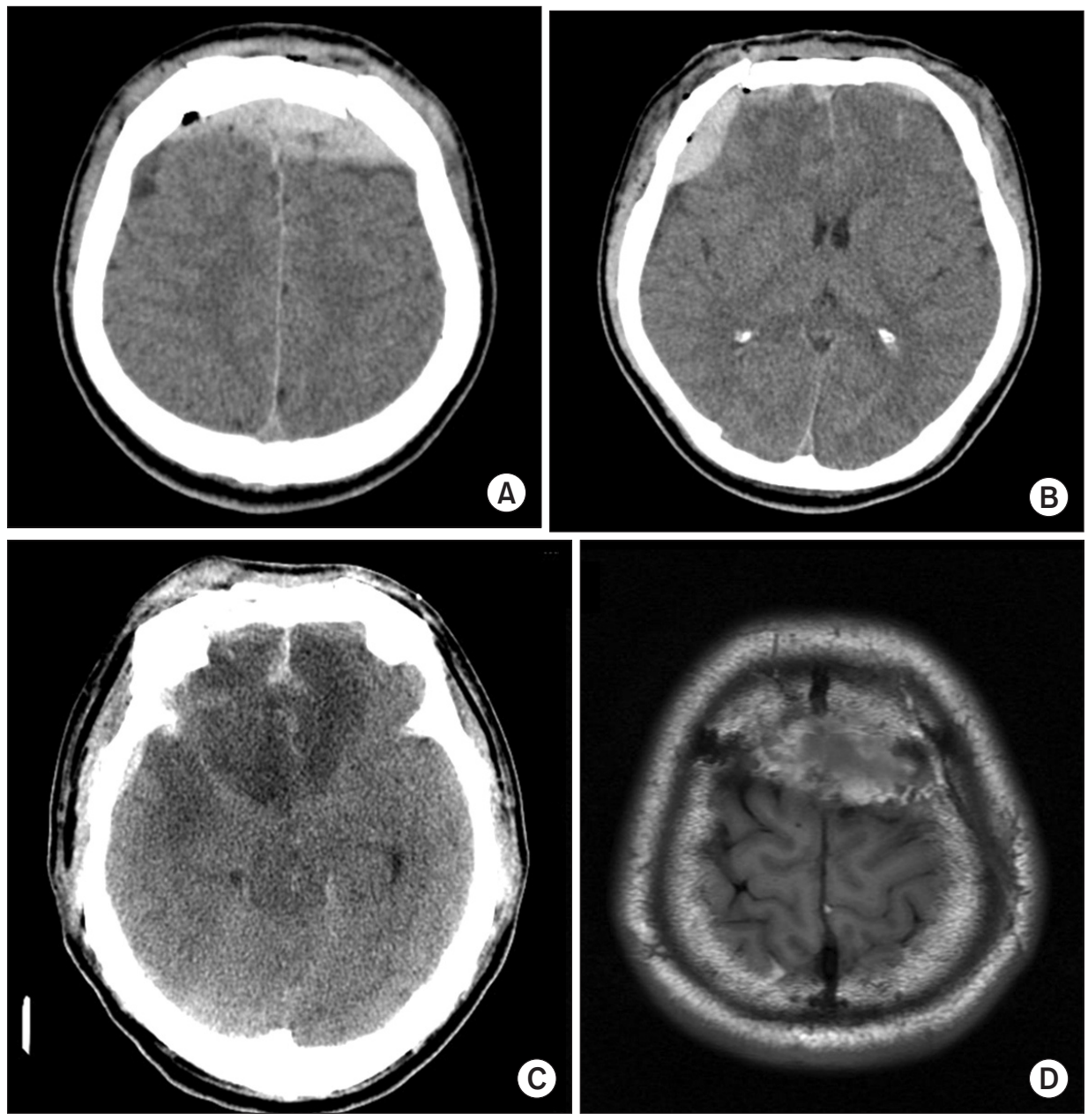

Fig. 1. Brain images of a 49-yearold man after a traffic accident. (A-C) Axial brain computed tomography images and (D) an axial T1 FLAIR brain magnetic resonance image show bifrontal convexity of epidural hematoma $(\mathrm{EDH})$, right frontal EDH, and hemorrhagic frontotemporal cerebral contusion. 


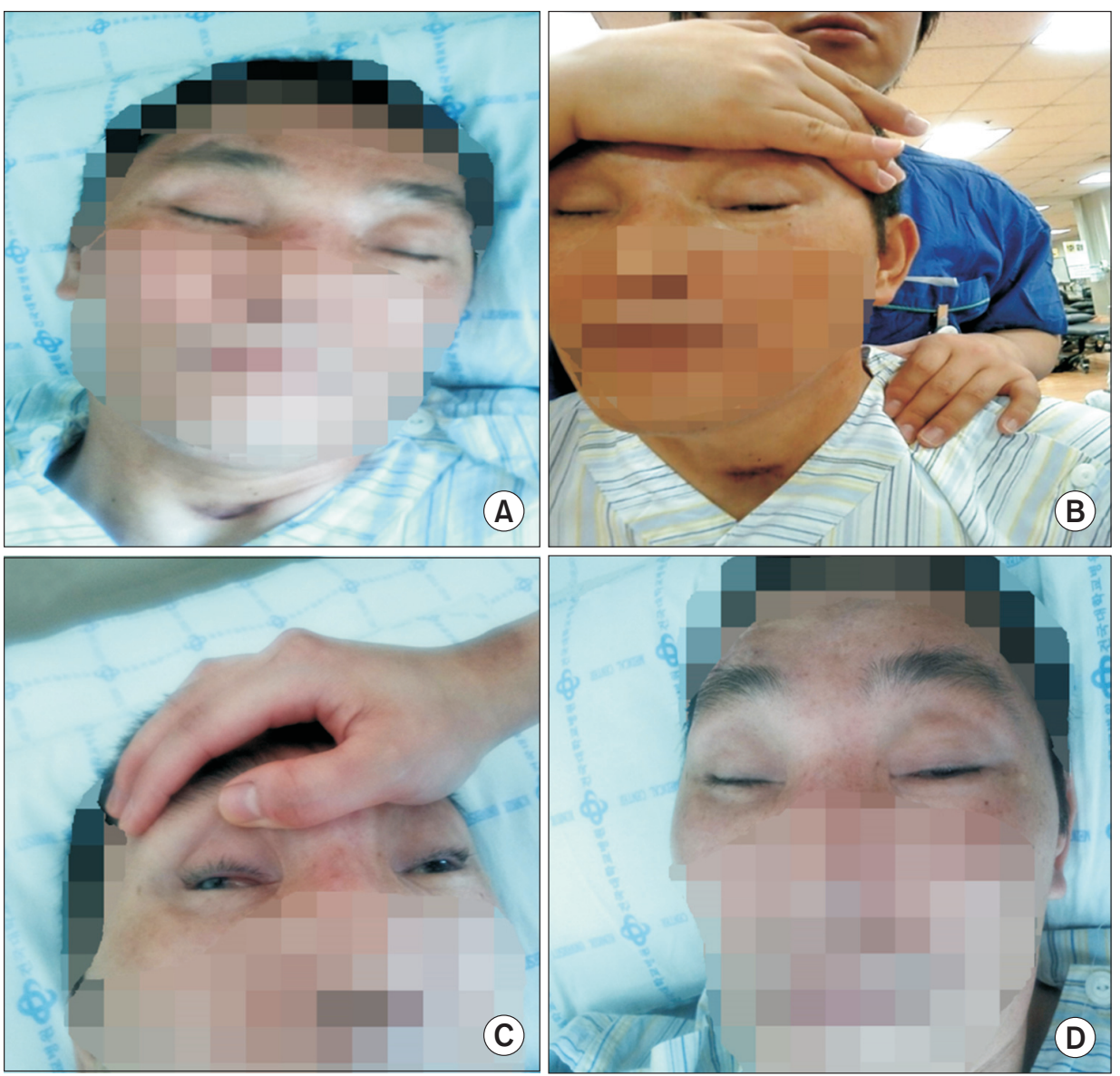

Fig. 2. Effectiveness of dopaminergic treatment. (A) Before the treatment, the patient could not open his eyes voluntarily. (B-D) After the dopaminergic treatment, spontaneous eyelid opening was observed. not improve. We planned on treating the patient with levodopa/benserazide $200 / 50 \mathrm{mg}$ twice a day with the informed consent of the patient. On the fifth day of the treatment, he started to open his eyes on command intermittently (Fig. 2). We confirmed no visual loss since he was able to recognize color and count fingers during spontaneous eye opening. With continued treatment, the interval between command and eye opening gradually shortened while the duration of spontaneous eye opening increased. This result was even more apparent when eating and undergoing physical training. We presumed that the dopaminergic treatment had a beneficial effect on AEO and tried to increase dose frequency from twice to three times daily to investigate a dose-dependent response. However, in the third day of the dosage modulation, aggressive behaviors emerged, and the treatment was withheld for three days. Even though the aggressiveness subsided shortly thereafter, the patient again exhibited difficulty in voluntary eye opening. After the resolution of the behavioral problem, the treatment was resumed twice a day. With AEO ameliorated, as evident in spontaneous eye opening during eating and physical training, the patient was discharged and has been closely followed up on an outpatient basis with the treatment of levodopa/benserazide $200 / 50 \mathrm{mg}$ twice a day. He is currently capable of voluntary eye opening that is sustained much longer than before being discharged.

\section{DISCUSSION}

Apraxia is the inability to execute learned skilled movements despite having the desire and the physical capacity to perform the movements. The underlying pathophysiology and anatomical site of the putative lesion are not well understood, but it has been linked to extrapyramidal diseases in a number of case reports. Goldstein and Cogan [1] described AEO in four patients with basal ganglia disease, and the subsequent reports emphasized the presence of this syndrome in extrapyramidal disorders. The frequent observation of AEO in extrapyramidal diseases suggests that the basal ganglia and their circuit could be the principal system involved. Schmidtke and 
Buttner-Ennever [8] mentioned that the cortex, extrapyramidal motor systems, and rostral midbrain structures may control levator palpebrae superioris (LPS) motor neuron activity. It is believed that AEO may involve an abnormality in the supranuclear control of voluntary eyelid elevation, which requires the activation of the LPS and the concurrent inhibition of orbicularis oculi activity [9]. Furthermore, Smith et al. [3] suggested that the presence of medial frontal hypometabolism and abnormal striatofrontal interactions without evidence of extrapyramidal diseases may have a possible role in the pathogenesis of AEO. This finding means that degenerative disorders associated with the frontal syndrome might be another cause of AEO.

This case report herein is a rare case presenting a patient with AEO suffered from traumatic brain injury without other extrapyramidal findings or signs of underlying parkinsonism. Based on the fact that the brain imaging shows lesions not in the basal ganglia but in both the frontal and right temporal lobes, we considered the possibility that the frontal lobe injury led to abnormal striatofrontal interaction; this conclusion was the rationale for the treatment with dopaminergic agents. On the fifth day of the treatment, spontaneous eye openings were noted, and its duration increased gradually. However, due to the emergence of aggressive behaviors after increasing the frequency, which resulted in difficulty of eye opening for a substantial period of time, the treatment was withheld. Based on this observation, we postulated that dopaminergic treatment would have some beneficial effect on improving AEO. In Korea, no reports on the effectiveness of dopaminergic treatment in AEO have been reported except for a case report of AEO emerging after subcortical infarction in right frontal lobe without a basal ganglia lesion [10]. In this regard, it is meaningful in that this case report herein marks the first report on the validity of the dopaminergic treatment.

Despite the intent to figure out the optimal dose to maintain eye opening, we could not evaluate dose-dependent response due to the manifestation of behavioral problems at a higher dose of levodopa/benserazide at $200 / 50 \mathrm{mg}$. The limitation of this study is that we could not only determine the effect of dosage adjustment but also the optimal treatment dose. Dewey and Maraganore [4] reported two cases of AEO successfully treated with levodopa and suggested the possibility that dysfunction of the basal ganglia circuits is the cause of AEO. Yamada et al. [5] reported on the remarkable effects of levodopa on a patient with AEO. Lee et al. [6] reported a patient with Parkinson disease developed AEO when the dosage of levodopa was reduced and improved dramatically when the dosage was increased. Umemura et al. [7] reported on two patients who suffered from disabling AEO after bilateral deep brain stimulation of the subthalamic nucleus. Their AEO improved by resuming the levodopa medication that had been discontinued after surgery. The dose of the dopaminergic medication would have some potential causality with AEO based on the aforementioned studies; the dysfunction of the basal ganglia structures or pathways presented as the possible pathogenesis of the AEO, such as abnormal striatofrontal interaction. This is the reason why we planned to use the levodopa/ benserazide $200 / 50 \mathrm{mg}$ in a patient with AEO without extrapyramidal disease. Further clinical and neuropathophysiological investigations are needed to confirm the beneficial effects and optimal therapeutic level of the dopaminergic treatment in AEO.

Conclusively, we report that AEO may occur after brain injury and that the levodopa/benserazide $200 / 50 \mathrm{mg}$ treatment is also beneficial in AEO patients without extrapyramidal diseases.

\section{CONFLICT OF INTEREST}

No potential conflict of interest relevant to this article was reported.

\section{REFERENCES}

1. Goldstein JE, Cogan DG. Apraxia of lid opening. Arch Ophthalmol 1965;73:155-9.

2. Riese W. Apraxia der lidoffnung (analyse einer bewegungsstorung). J Psychol Neurol Lpz 1930;40:347-55.

3. Smith D, Ishikawa T, Dhawan V, Winterkorn JS, Eidelberg D. Lid opening apraxia is associated with medial frontal hypometabolism. Mov Disord 1995;10:341-4.

4. Dewey RB Jr, Maraganore DM. Isolated eyelid-opening apraxia: report of a new levodopa-responsive syndrome. Neurology 1994;44:1752-4.

5. Yamada S, Matsuo K, Hirayama M, Sobue G. The effects of levodopa on apraxia of lid opening: a case report. Neurology 2004;62:830-1. 
6. Lee KC, Finley R, Miller B. Apraxia of lid opening: dose-dependent response to carbidopa-levodopa. Pharmacotherapy 2004;24:401-3.

7. Umemura A, Toyoda T, Yamamoto K, Oka Y, Ishii F, Yamada K. Apraxia of eyelid opening after subthalamic deep brain stimulation may be caused by reduction of levodopa. Parkinsonism Relat Disord 2008;14:655-7.

8. Schmidtke K, Buttner-Ennever JA. Nervous control of eyelid function: a review of clinical, experimental and pathological data. Brain 1992;115 Pt 1:227-47.

9. Esteban A, Traba A, Prieto J. Eyelid movements in health and disease: the supranuclear impairment of the palpebral motility. Neurophysiol Clin 2004;34:315.

10. Lee SS, Lee HS. Can subcortical infarction cause apraxia of eyelid opening? J Clin Neurosci 2011;18:1399400 . 